\title{
TITLE: SYNTHETIC DNA STANDARDS FOR GENETIC SCREENING FOR MH/CCD
}

\author{
AUTHORS: ChunHua Yu MD*, Julian C.P. Loke MBBS BMedSc ${ }^{*}$, and David H MacLennan PhD \\ AFFILIATION: *Malignant Hyperthermia Investigation Unit, Department of Anesthesia \& Pain \\ Management, University of Toronto, 200 Elizabeth Street, Room 3ES-403, Toronto, ON, M5G 2C4 \\ ${ }^{\#}$ Banting and Best Department of Medical Research, University of Toronto 112 College St, Room 305, \\ Toronto, ON, M5G 1L6
}

INTRODUCTION: To date, 96 mutations in the Ryanodine Receptor Isoform 1 gene (RYR1) have been reported to be associated with malignant hyperthermia $(\mathrm{MH})$ and central core disease (CCD) (1). These mutations are located over a span of 160 kilobase of genomic DNA and are located in 32 exons. Although a few mutations occur with slightly higher frequency, most of the mutations are private to individual families, and are extremely rare. In this project, we developed a method to synthesize DNA with all currently known MH/CCD mutations, intended for use as a positive control in future genetic screening.

METHODS: Reverse electronic PCR (reverse e-PCR) was used to search the human genome database [9606] for suspect or causal mutations of MH/CCD. Each mutation site was uniquely identified and flanking DNA sequences downloaded into a custom local database programmed in Microsoft Excel. Each of these flanking regions was divided into overlapping cis-trans fragments that contained complementary 10 base sticky ends. The resultant fragments were synthesized commercially.

Positive control double strand DNA fragments were synthesized by polymerase chain reactions (PCR). Correct fragment assembly was verified by sensitivity to digestion with Uracil-DNA glycosylase (UNG) that is only present with uracil and not thymidine incorporation (Fig. 1). Following a subsequent round of PCR amplification, products were processed for cycle sequencing.

RESULTS: Sequences within 30 exons of RYRI and 1 exon of CACNA1S and 1 exon of CPT2 were identified and downloaded by reverse e-PCR. Ninety-six mutations were synthetically incorporated, then each sequence was divided into 2 to 8 fragments according to length, so that individual template oligonucleotides were between 25-86 bases. Average length of the single exon fragments is 127 bases, with standard deviation (SD) of 43 bases. $\% \mathrm{GC}$ is $62.79 \%$ with $\mathrm{SD}$ of $6.19 \%$.

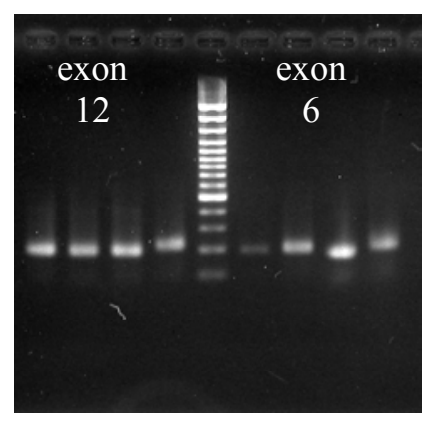

DISCUSSION: Using a bioinformatics approach, synthetic DNA standards were constructed using in vitro synthesis and PCR. These positive controls contain all currently known mutations, paving the way for genetic screening for $\mathrm{MH} / \mathrm{CCD}$.

REFERENCES:

1. Br J Anaesth. 2001 Feb; 86(2): 283-7 